\title{
An Analytic Representation of Quantum Field Theory
}

\author{
D. K. Saraswati and J. G. Valatin \\ Department of Physics, Queen Mary College, University of London
}

Received December 2, 1968

\begin{abstract}
The connection between a space of quadratically integrable functions of real variables $q$ and a Hilbert space of analytic functions of complex variables $z$ established by BARGMANN is used to introduce quantised field operators for which the $\delta$-functions of the commutation relations in $q$-space are replaced by analytic kernel functions in $z$-space, and a reference to distributions can be avoided. BARGMANN's representation is first somewhat modified, so that the derivative terms in the field equations retain their form in the new representation. Local interaction terms in $q$-space obtain a non-local appearance in $z$-space. The transition to a 4-dimensional formulation in $z$-space has to resort to a Euclidean metric. The equations can be derived directly by starting from an action integral in $z$-space, and applying a variational calculus in which variations are restricted to analytic functions. Explicit analytic expressions are given for free field propagators.
\end{abstract}

\section{Introduction}

BARGMANN established in detail a correspondence between a space of quadratically integrable functions of real variables and a Hilbert space of analytic functions [1]. If one associates complex variables with creation operators of harmonic oscillators, the connection of analytic functions of these variables with wave functions in configuration space has been known for some time, but the scalar product of the analytic function space in BARGMANN's representation and the related kernel functions were new in quantum mechanics, and may still reveal unexplored relationships.

Bargmand [2], Glauber [3], Klauder [4] and others have attempted a generalisation of the related concepts to the case of an infinite number of complex variables connected with the description of a quantised boson field in terms of infinitely many oscillator components. This involves the interesting concept of "coherent states", but is not the line to be followed in the present work.

The rather different approach to connect spaces of analytic functions with operators of quantum field theory to be followed here, is based on the simple feature of Bargmann's representation that the $\delta$-function kernel of the unit operator is replaced by a reproducing kernel which is an analytic function of its variables. If one succeeds in replacing the $\delta$-functions in the commutation relations of quantised field operators by 
analytic functions, and can avoid a reference to distributions and to the related improper field operators, the advantages of analytic functions may provide a powerful tool to attack some of the mathematical problems of quantum field theory.

As will be seen, in the framework of non-relativistic quantum theory, the second quantised operators as functions of complex variables $z$ will have such analytic equal time commutation relations, and will be proper creation and annihilation operators of normalisable single particle states. Similar relationships can be established for relativistic fields, if one treats the time variable on a separate basis, and performs the transformations to complex variables only with respect to the space coordinates $q$.

If one aims at a symmetric 4-dimensional form of the equations in $z$-space, an attempt of a direct transformation of the $q$-space equations forces one to adopt a Euclidean metric, because of the sign of exponents in Gaussian integrations. Schwinger [5], Nakano [6], Symanzik [7] and others have discussed field theory in Euclidean space, the connection of which with field theory in Minkowski space-time can be established by means of analytic continuations. According to SrmanzIK, the existence of the related Euclidean quantum field theory is a necessary condition for the existence of solutions of a local theory in Minkowski space. Restrictions on Euclidean field theories in $z$-space derived from analyticity requirements would therefore have consequences in Minkowski space too.

Bargmann's transformation to z-space is adopted in a somewhat modified form, in which it appears more directly as a Gaussian averaging process, the spread of which in $q$-space is exhibited explicitly by a length $a$ which can be chosen arbitrarily. Local interactions in $q$-space lead in $z$-space to field equations and Green's function equations of a non-local appearance, with an interaction kernel $W$. The kernel $W$ itself depends on the length $a$, but this is only a feature of the representation. As long as the action integral in $q$-space is independent of $a$ and the averaging process, all physical conclusions will remain independent of it. One could explore whether this representation does not suggest some modification of the theory in which a fundamental length of physical significance appears.

\section{Analytic Single Particle Wave Functions and Kernels}

According to BARGMANN [1], quadratically integrable functions $\varphi(q)$ of a real variable $q$ can be transformed into analytic functions $\hat{f}(Z)$ of a complex variable $Z$ by means of a transformation

$$
\hat{f}(Z)=\int d q \hat{A}(Z, q) \varphi(q)
$$


with

$$
\hat{A}(Z, q)=\pi^{-1 / 4} \exp \left[-\frac{1}{2}\left(Z^{2}+q^{2}\right)+\sqrt{2} Z q\right] .
$$

The notation has been slightly changed, so that the original symbols can be used with the somewhat modified meaning given below. The scalar product of two functions $\hat{f}(Z), \hat{f}^{\prime}(Z)$ of the new function space can be defined by the integral

$$
\left(\hat{f}, \hat{f}^{\prime}\right)=\int d \hat{\mu}(Z) \overline{\hat{f}(Z)} \hat{f}^{\prime}(Z)
$$

where

$$
d \hat{\mu}(Z)=\frac{d X d Y}{\pi} \exp [-Z \bar{Z}]
$$

and $Z=X+i Y, Z \bar{Z}=X^{2}+Y^{2}$. This is equal to the scalar product

$$
\left(\varphi, \varphi^{\prime}\right)=\int d q \varphi^{*}(q) \varphi^{\prime}(q)
$$

of the two corresponding functions $\varphi(q), \varphi^{\prime}(q)$.

With some care [1], the inverse operation

$$
\varphi(q)=\int d \hat{\mu}(Z) \hat{A}(\bar{Z}, q) \hat{f}(Z)
$$

can also be defined, and the kernel $\hat{A}(Z, q)$ establishes a one-to-one correspondence between the $L_{2}$ space of functions $\varphi(q)$ and a Hilbert space of analytic functions $\hat{f}(Z)$. One finds

$$
\begin{aligned}
\int d \hat{\mu}(Z) \hat{A}(Z, q) \hat{A}\left(\bar{Z}, q^{\prime}\right) & =\delta\left(q-q^{\prime}\right), \\
\int d q \hat{A}(Z, q) \hat{A}\left(\bar{Z}^{\prime}, q\right) & =\exp \left[Z \bar{Z}^{\prime}\right],
\end{aligned}
$$

and $\exp \left[Z \bar{Z}^{\prime}\right]$ plays the role of a reproducing kernel,

$$
\int d \hat{\mu}(Z) \exp \left[Z \bar{Z}^{\prime}\right] \hat{f}\left(Z^{\prime}\right)=\hat{f}(Z) \text {. }
$$

The transformation is closely related to the functions

$$
\varphi_{x ; k}(q)=\left(\pi a^{2}\right)^{-1 / 4} \exp [i k q] \exp \left[-\frac{(q-x)^{2}}{2 a^{2}}\right],
$$

which represent wave packets of average position $x$ and average momentum $\hbar k$. For wave functions of this form the inequality $\triangle q \cdot \triangle p \geqq \frac{1}{2} \hbar$ of the uncertainty principle for the mean square deviation of the coordinate $q$ and momentum $p=-i \hbar d / d q$ becomes an equality [8], and they are eigenfunctions of the operator $q+i a^{2} p / \hbar$ with eigenvalues $\bar{z}=x+i a^{2} k$. They satisfy the relationship

$$
\int \frac{d x d(-k)}{2 \pi} \varphi_{x ; k}(q) \varphi_{x ; k}^{*}\left(q^{\prime}\right)=\delta\left(q-q^{\prime}\right)
$$

and lead to a continuous decomposition [4]

$$
\varphi(q)=\int \frac{d x d(-k)}{2 \pi} \varphi_{x ; k}(q) \int d q^{\prime} \varphi_{x ; k}^{*}\left(q^{\prime}\right) \varphi\left(q^{\prime}\right)
$$

of the functions $\varphi(q)$.

18 Commun.math. Phys., Vol. 12 
The complex variable $z$ to be used will be related to the parameters of the wave packet ( $3 a$ ) by

$$
\begin{aligned}
& z=x-i a^{2} k, \quad \bar{z}=x+i a^{2} k, \\
& x=\frac{1}{2}(z+\bar{z}), \quad k=\frac{i}{2 a^{2}}(z-\bar{z}) .
\end{aligned}
$$

BARGMANN's choice of the transformation kernel corresponds to a value $a=1 / \sqrt{2}$ of the positive real constant $a$ which describes the spread of the Gaussian wave packet. With

one can write

$$
Z=\frac{z}{\sqrt{2} a}
$$

$$
\hat{A}(Z, q)=\exp \left[\frac{x^{2}}{2 a^{2}}\right] \varphi_{x ; k}^{*}(q) \exp \left[-\frac{z^{2}}{4 a^{2}}\right] .
$$

The analytic functions $f(z)$ to be considered will be related to the functions $\hat{f}(Z)$ by

With

$$
f(z)=\exp \left[-\frac{1}{2} Z^{2}\right] \hat{f}(Z)
$$

$$
A(z, q)=\exp \left[-\frac{1}{2} Z^{2}\right] \hat{A}(Z, q)
$$

they can be obtained directly from the functions $\varphi(q)$ by the transformation

$$
f(z)=\int d q A(z, q) \varphi(q)
$$

The factor $\exp \left[-\frac{1}{2} Z^{2}\right]$ in the relationships $(5 \mathrm{a})(5 \mathrm{~b})$ is chosen to make the transformation of differential operators simpler. The kernel $A(z, q)$ can also be written in the form

$$
A(z, q)=\left(\pi a^{2}\right)^{-1 / 4} \exp \left[-\frac{(z-q)^{2}}{2 a^{2}}\right] .
$$

The scalar product of two functions $f(z), f^{\prime}(z)$ in the Hilbert space of analytic functions $f(z)$ can be defined as

with

$$
\left(f, f^{\prime}\right)=\int d \mu(z) \overline{f(z)} f^{\prime}(z)
$$

$$
\begin{aligned}
d \mu(z) & =\frac{d x d(-k)}{2 \pi} \exp \left[\left(\frac{z-\bar{z}}{2 a}\right)^{2}\right] \\
& =\frac{d x d(-k)}{2 \pi} \exp \left[-a^{2} k^{2}\right] .
\end{aligned}
$$

This is the same as the product (1c) defined for functions $\hat{f}(Z), \hat{f}^{\prime}(Z)$ which are related to $f(z), f^{\prime}(z)$ by the relationship (5a), and is equal to the scalar product $(1 \mathrm{e})$ of the functions $\varphi(q), \varphi^{\prime}(q)$. 
The relationships $(2 \mathrm{~b})(2 \mathrm{c})$ take the form

$$
\begin{aligned}
& \int d \mu(z) A(z, q) A\left(\bar{z}, q^{\prime}\right)=\delta\left(q-q^{\prime}\right) \\
& \int d q A(z, q) A\left(\bar{z}^{\prime}, q\right)=\exp \left[-\frac{\left(z-\bar{z}^{\prime}\right)^{2}}{4 a^{2}}\right] .
\end{aligned}
$$

The reproducing unit kernel is

and one has

$$
U\left(z-\bar{z}^{\prime}\right)=\exp \left[-\frac{\left(z-\bar{z}^{\prime}\right)^{2}}{4 a^{2}}\right]
$$

$$
\int d \mu\left(z^{\prime}\right) U\left(z-\bar{z}^{\prime}\right) f\left(z^{\prime}\right)=f(z) .
$$

The correspondence between operators of the two function spaces is defined by the unitary mapping $A$. Any integral operator kernel $K\left(q, q^{\prime}\right)$ of an operator acting on functions $\varphi(q)$ corresponds in the $z$-representation to a kernel

$$
K\left(z, \bar{z}^{\prime}\right)=\iint d q d q^{\prime} A(z, q) K\left(q, q^{\prime}\right) A\left(\bar{z}^{\prime}, q^{\prime}\right) .
$$

The $\delta$-function kernel $\delta\left(q-q^{\prime}\right)$ of the unit operator is transformed according to $(7 \mathrm{~b}, \mathrm{c})$ into the unit kernel $U\left(z-\bar{z}^{\prime}\right)$. One finds the relationships

$$
\begin{aligned}
\delta\left(q-q^{\prime}\right) & \leftrightarrow U\left(z-\bar{z}^{\prime}\right) \\
q \delta\left(q-q^{\prime}\right) & \leftrightarrow \frac{1}{2}\left(z+\bar{z}^{\prime}\right) U\left(z-\bar{z}^{\prime}\right) \\
\frac{\hbar}{i} \frac{\partial}{\partial q} \delta\left(q-q^{\prime}\right) & \leftrightarrow \frac{i \hbar}{2 a^{2}}\left(z-\bar{z}^{\prime}\right) U\left(z-\bar{z}^{\prime}\right) \\
V(q) \delta\left(q-q^{\prime}\right) & \leftrightarrow\left(\pi a^{2}\right)^{-1 / 2} \int d q \exp \left[-\frac{1}{a^{2}}\left(q-\frac{z+\bar{z}^{\prime}}{2}\right)^{2}\right] V(q) \cdot U\left(z-\bar{z}^{\prime}\right)
\end{aligned}
$$

In the last correspondence, the factor multiplying the unit kernel $U\left(z-\bar{z}^{\prime}\right)$ is, for $z=z^{\prime}$ and with $\frac{1}{2}(z+\bar{z})=x$, a Gaussian average of $V(q)$. The relationships $(8 \mathrm{c}, \mathrm{d})$ can also be written in the operator form

$$
\begin{aligned}
& q \leftrightarrow\left(z+a^{2} \frac{\partial}{\partial z}\right), \\
& p=\frac{\hbar}{i} \frac{\partial}{\partial q} \leftrightarrow \frac{\hbar}{i} \frac{\partial}{\partial z} .
\end{aligned}
$$

The simple transformation $(9 \mathrm{~b})$ of the differential operators ensures at the same time a very simple connection between Fourier representations of the functions $\varphi(q)$ and $f(z)$. In writing

$$
\begin{aligned}
& \varphi(q)=\frac{1}{2 \pi} \int d K \exp [i K q] \tilde{\varphi}(K), \\
& f(z)=\frac{1}{2 \pi} \int d K \exp [i K z] \tilde{f}(K),
\end{aligned}
$$


the transformation $(6 a, b)$ gives after elementary integrations

$$
\tilde{f}(K)=\left(4 \pi a^{2}\right)^{1 / 4} \exp \left[-\frac{1}{2} a^{2} K^{2}\right] \tilde{\varphi}(K) .
$$

Functions $\varphi\left(q_{1}, q_{2}, q_{3}\right)$ of three real variables are transformed into functions $f\left(z_{1}, z_{2}, z_{3}\right)$ by means of a kernel

$$
A^{(3)}(z, q)=A\left(z_{1}, q_{1}\right) A\left(z_{2}, q_{2}\right) A\left(z_{3}, q_{3}\right) \text {. }
$$

In the space of analytic functions $f\left(z_{1}, z_{2}, z_{3}\right)$, the scalar product is defined by integrations with $d^{3} \mu(z)=d \mu\left(z_{1}\right) d \mu\left(z_{2}\right) d \mu\left(z_{3}\right)$ and the reproducing unit kernel is

$$
U^{(3)}\left(z-\bar{z}^{\prime}\right)=U\left(z_{1}-\bar{z}_{1}^{\prime}\right) U\left(z_{2}-\bar{z}_{2}^{\prime}\right) U\left(z_{3}-\bar{z}_{3}^{\prime}\right) .
$$

BARGMANN actually establishes the corresponding relationships for functions of $n$-variables, and thus for the $3 n$ variables resulting after a transformation of the wave functions of configuration space.

\section{Second Quantised Operators}

For non-relativistic particles, in terms of a complete system of single particle states $\varphi_{\varkappa}(q)$ and corresponding creation and annihilation operators $a_{x}, a_{x}^{+}$, the quantised field operators $\Psi[q], \Psi^{*}\left[q^{\prime}\right]$ can be written in the form

$$
\Psi[q]=\sum_{x} \varphi_{\varkappa}(q) a_{\varkappa}^{+}, \quad \Psi^{*}\left[q^{\prime}\right]=\sum_{\varkappa} \varphi_{\varkappa}^{*}\left(q^{\prime}\right) a_{\varkappa} .
$$

In the case of fermions, the field operators satisfy the anticommutation relations

$$
\begin{gathered}
\left\{\Psi[q], \Psi^{*}\left[q^{\prime}\right]\right\}_{+}=\delta^{(3)}\left(q-q^{\prime}\right), \\
\left\{\Psi[q], \Psi\left[q^{\prime}\right]\right\}_{+}=0, \\
\left\{\Psi^{*}\left[q^{\prime}\right], \Psi^{*}[q]\right\}_{+}=0 .
\end{gathered}
$$

The variables $q$ stand for the three space coordinates. Internal variables, like spin, should be included, but these will not enter into the transformations.

In applying the same transformation

$$
f_{\varkappa}(z)=\int d^{3} q A^{(3)}(z, q) \varphi_{\varkappa}(q)
$$

to all single particle wave functions, the operators (12a) are transformed into the operators

$$
\begin{aligned}
\Psi(z) & =\int d^{3} q A^{(3)}(z, q) \Psi[q], \\
\Psi^{*}\left(\bar{z}^{\prime}\right) & =\int d^{3} q^{\prime} \Psi^{*}\left[q^{\prime}\right] A^{(3)}\left(\bar{z}^{\prime}, q^{\prime}\right),
\end{aligned}
$$

which can be expanded as

$$
\Psi(z)=\sum_{x} f_{x}(z) a_{\varkappa}^{+}, \quad \Psi^{*}\left(\bar{z}^{\prime}\right)=\sum_{x} \overline{f_{x}(z)} a_{\varkappa}
$$


in terms of the same creation and annihilation operators $a_{\varkappa}, a_{x}^{+}$. The new field operators depend on the complex variables $z$ and satisfy the anticommutation relations

$$
\begin{gathered}
\left\{\Psi(z), \Psi^{*}\left(\bar{z}^{\prime}\right)\right\}_{+}=U^{(3)}\left(z-\bar{z}^{\prime}\right), \\
\left\{\Psi(z), \Psi\left(z^{\prime}\right)\right\}_{+}=0, \quad\left\{\Psi^{*}\left(\bar{z}^{\prime}\right), \Psi^{*}(\bar{z})\right\}_{+}=0 .
\end{gathered}
$$

These follow by transforming the relations $(12 b)$. The $\delta$-function in these is replaced by the unit kernel $U^{(3)}\left(z-\bar{z}^{\prime}\right)$ which is an analytic function of the variables $z$ and $\bar{z}^{\prime}$.

Whereas $\Psi[q], \Psi *\left[q^{\prime}\right]$ are improper operators related to distributions, apart from a normalisation factor, $\Psi^{*}(\bar{z})$ is the creation operator of the single particle state $\varphi_{x ; k}(q)$ which corresponds in 3 -dimensions to the wave function $(3 \mathrm{a})$.

The number operator can be transformed into the form

$$
N=\int d^{3} \mu(z) \Psi^{*}(\bar{z}) \Psi(z) .
$$

If one includes the weight factor in the definition of a phase space density operator

$$
\tilde{\varrho}(z, \bar{z})=\tilde{\varrho}(x ; k)=\exp \left[\left(\frac{z-\bar{z}}{2 a}\right)^{2}\right] \Psi *(\bar{z}) \Psi(z),
$$

one can write

$$
N=\int \frac{d^{3} x d^{3}(-k)}{(2 \pi)^{3}} \tilde{\varrho}(x ; k) .
$$

In contrast to density operators in $q$-space, $\tilde{\varrho}(z, \bar{z})$ is a proper operator, and one finds

$$
\tilde{\varrho}(z, \bar{z}) \varrho(z, \bar{z})=\tilde{\varrho}(z, \bar{z}) \text {. }
$$

That is $\tilde{\varrho}(z, \bar{z})$ is a projection operator, with eigenvalues 1 and 0 and all its expectation values are non-negative.

The connection between one particle operators in the $q$ and $z$ representations is given by the correspondence

$$
\begin{aligned}
\iint d^{3} q d^{3} q^{\prime} \Psi^{*}[q] K\left(q, q^{\prime}\right) \Psi\left[q^{\prime}\right] \\
\leftrightarrow \iint d^{3} \mu(z) d^{3} \mu\left(z^{\prime}\right) \Psi^{*}(\bar{z}) \mathscr{K}\left(z, \bar{z}^{\prime}\right) \Psi\left(z^{\prime}\right),
\end{aligned}
$$

where the kernels $K$ and $\mathscr{K}$ are related to each other according to the transformation (8a).

The total momentum operator

$$
P_{j}=\int d^{3} \mu(z) g_{j}(z, \bar{z}), \quad j=1,2,3
$$

can be expressed in terms of the momentum density operator

$$
g_{j}(z, \bar{z})=\frac{\hbar}{2 i}\left\{\Psi *(\bar{z}) \frac{\partial}{\partial z_{j}} \Psi(z)-\frac{\partial \Psi^{*}(\bar{z})}{\partial \bar{z}_{j}} \Psi(z)\right\} .
$$

In analogy to $(14 \mathrm{~b})$, one could define a phase space momentum density $18 \mathrm{a}$ 
operator $\tilde{g}_{j}(x ; k)=\exp \left[-a^{2} k^{2}\right] g_{j}$. In considering $z$ and $\bar{z}$ as independent variables, one can write

$$
g_{j}(z, \bar{z})=\frac{\hbar}{2 i}\left(\frac{\partial}{\partial z_{j}}-\frac{\partial}{\partial \bar{z}_{j}}\right)(\Psi *(\bar{z}) \Psi(z)),
$$

so that the phase space density operator determines the momentum density. Note that

$$
\frac{1}{i}\left(\frac{\partial}{\partial z_{j}}-\frac{\partial}{\partial \bar{z}_{j}}\right)=\frac{1}{a^{2}} \frac{\partial}{\partial k_{j}} .
$$

With the correspondence $\nabla_{q}^{2} \leftrightarrow \Gamma_{z}^{2}$, the kinetic energy of a system is described by the operator

$$
T=-\frac{\hbar^{2}}{2 m} \int d^{3} \mu(z) \Psi^{*}(\bar{z}) \nabla_{z}^{2} \Psi(z) .
$$

A two-body interaction potential $V\left(q_{(1)}, q_{(2)}\right)$ corresponds in general to a non-local kernel $\mathscr{V}\left(z_{(1)}, z_{(2)} ; \bar{z}_{(1)}^{\prime}, \bar{z}_{(2)}^{\prime}\right)$ in $z$-space, and to an interaction energy operator

$$
\begin{aligned}
V=\frac{1}{2} \int & \cdots \int d^{3} \mu\left(z_{(1)}\right) d^{3} \mu\left(z_{(2)}\right) d^{3} \mu\left(z_{(1)}^{\prime}\right) d^{3} \mu\left(z_{(2)}^{\prime}\right) \\
& \cdot \mathscr{V}\left(z_{(1)}, z_{(2)} ; \bar{z}_{(1)}^{\prime}, \bar{z}_{(2)}^{\prime}\right) \Psi^{*}\left(\bar{z}_{(1)}\right) \Psi^{*}\left(\bar{z}_{(2)}\right) \Psi\left(z_{(1)}^{\prime}\right) \Psi\left(z_{(2)}^{\prime}\right) .
\end{aligned}
$$

The Hamiltonian of a non-relativistic system of fermions, in the absence of external fields, can be described by the operator $H=T+V$. One could attempt to find models with an interaction which is simpler in the $z$-representation.

\section{Field Equations. Green's Functions}

As an example, a 4 -component spin $1 / 2$ fermion field $\Psi, \bar{\Psi}$ in interaction with a neutral scalar boson field $\Phi$ will be considered in the following, with a local interaction density in $q$-space of the form $g \Phi[q] \bar{\Psi}[q] \Psi[q]$. The canonical formulation of relativistic field theory, with a Hamiltonian and equal-time commutation relations, imitates concepts of a non-relativistic theory. In the same spirit, in a given reference system, one can attempt first to transform the fields to the $z$-representation only with respect to the three space coordinates. The equations will not have an invariant form, but their content will be equivalent to the formulation in $q$-space.

The Hamiltonian of the model considered can be obtained in terms of $z$-space fields by a straightforward transformation of the $q$-space Hamiltonian, in the form

$$
\begin{aligned}
H= & \int d^{3} \mu(z) \bar{\Psi}(\bar{z}, t)\left(-\boldsymbol{\gamma} \cdot i \boldsymbol{V}_{z}+m\right) \Psi(z, t) \\
& +\frac{1}{2} \int d^{3} \mu(z)\left\{\frac{\partial \Phi(\bar{z}, t)}{\partial t} \frac{\partial \Phi(z, t)}{\partial t}-\Phi(\bar{z}, t) \nabla_{z}^{2} \Phi(z, t)+\varkappa^{2} \Phi(\bar{z}) \Phi(z)\right\} \\
& +g \iiint d^{3} \mu(z) d^{3} \mu\left(z^{\prime}\right) d^{3} \mu\left(z^{\prime \prime}\right) W^{(3)}\left(\bar{z}, z^{\prime}, \bar{z}^{\prime \prime}\right) \Phi(z, t) \bar{\Psi}\left(\bar{z}^{\prime}, t\right) \Psi\left(z^{\prime \prime}, t\right) .
\end{aligned}
$$


The transformations from $q$-space field operators are given by the Eqs. (13b). The real field $\Phi[q, t]$ can be transformed by the analogous equations either into $\Phi(z, t)$ or into $\Phi(\bar{z}, t)$, and the integrands of the terms of (18a) could be symmetrised with respect to $z$ and $\bar{z}$. Units with $\hbar=1, c=1$, will be adopted. The local $q$-space interaction appears in a non-local form in $z$-space, described by the kernel

$$
W^{(3)}\left(z, z^{\prime}, z^{\prime \prime}\right)=\int d^{3} q A^{(3)}(z, q) A^{(3)}\left(z^{\prime}, q\right) A^{(3)}\left(z^{\prime \prime}, q\right),
$$

which is symmetric in $z, z^{\prime}, z^{\prime \prime}$. A straightforward integration gives

$$
W^{(3)}\left(z, z^{\prime}, z^{\prime \prime}\right)=\left(\pi\left(\frac{3}{2} a\right)^{2}\right)^{-3 / 4} \exp \left[-\frac{\left(z-z^{\prime}\right)^{2}+\left(z^{\prime}-z^{\prime \prime}\right)^{2}+\left(z^{\prime \prime}-z\right)^{2}}{6 a^{2}}\right] \text {. }
$$

The equal-time canonical commutation and anticommutation relations are transformed into

$$
\begin{gathered}
\left\{\Psi(z, t), \bar{\Psi}\left(\bar{z}^{\prime}, t\right)\right\}_{+}=\gamma_{0} U^{(3)}\left(z-\bar{z}^{\prime}\right), \\
\left\{\Psi(z, t), \Psi\left(z^{\prime}, t\right)\right\}_{+}=0, \quad\left\{\bar{\Psi}(\bar{z}, t), \bar{\Psi}\left(\bar{z}^{\prime}, t\right)\right\}_{+}=0, \\
{\left[\Phi(z, t), \frac{\partial \Phi\left(\bar{z}^{\prime}, t\right)}{\partial t}\right]=i U^{(3)}\left(z-\bar{z}^{\prime}\right),} \\
{\left[\Phi(z, t), \Phi\left(z^{\prime}, t\right)\right]=0 .}
\end{gathered}
$$

The field equations result from

$$
\frac{\partial}{\partial t} \Psi=i[H, \Psi], \quad \frac{\partial}{\partial t} \bar{\Psi}=i[H, \bar{\Psi}], \quad \frac{\partial}{\partial t} \Phi=i[H, \Phi]
$$

in the form

$$
\begin{aligned}
& \left(-\gamma_{0} i \frac{\partial}{\partial t}-\boldsymbol{\gamma} \cdot i \boldsymbol{V}_{z}+m\right) \Psi(z, t) \\
& \quad+g \iint d^{3} \mu\left(z^{\prime}\right) d^{3} \mu\left(z^{\prime \prime}\right) W^{(3)}\left(z, \bar{z}^{\prime}, \bar{z}^{\prime \prime}\right) \Phi\left(z^{\prime}, t\right) \Psi\left(z^{\prime \prime}, t\right)=0 \\
& \bar{\Psi}(\bar{z}, t)\left(\gamma_{0} i \frac{\partial}{\partial t}+\boldsymbol{\gamma} \cdot i \boldsymbol{V}_{z}+m\right) \\
& \quad+g \iint d^{3} \mu\left(z^{\prime}\right) d^{3} \mu\left(z^{\prime \prime}\right) \bar{\Psi}\left(\bar{z}^{\prime \prime}, t\right) \Phi\left(z^{\prime}, t\right) W^{(3)}\left(\bar{z}, \bar{z}^{\prime}, z^{\prime \prime}\right)=0 \\
& \left(\frac{\partial^{2}}{\partial t^{2}}-\nabla_{z}^{2}+x^{2}\right) \Phi(z, t) \\
& \quad-g \iint d^{3} \mu\left(z^{\prime}\right) d^{3} \mu\left(z^{\prime \prime}\right) W^{(3)}\left(z, z^{\prime}, \bar{z}^{\prime \prime}\right) \Psi\left(\bar{z}^{\prime}, t\right) \Psi\left(z^{\prime \prime}, t\right)=0 .
\end{aligned}
$$

The field $\Phi(\bar{z}, t)$ satisfies Eq. (20c) with $z$ replaced by $\bar{z}$. A direct transformation of the $q$-space field equations into $z$-space would give the same Eqs. $(20 a, b, c)$.

If one wishes to make a similar transformation for the time variable $t$, one finds that the exponents of the Gaussian factors of the transformation $(6 a, b)$, of the metric $(6 \mathrm{~d})$ and the unit function $(7 \mathrm{c})$ have all to be taken with the sign given in these expressions, and the opposite sign would 
lead to divergent integrals. One would then obtain total exponents of the type $q_{1}^{2}+q_{2}^{2}+q_{3}^{2}+t^{2}$ and $z_{1}^{2}+z_{2}^{2}+z_{3}^{2}+z_{0}^{2}$, with no simple invariance properties. Instead, one can attempt a transition to $z$-space within a Euclidean metric and connect the results with physical conclusions in a Minkwoski metric by means of analytic continuation. In writing $q_{4}=i$, and considering $q_{4}$ as a real variable, the transformation

$$
\Psi(z)=\Psi\left(z ; z_{4}\right)=\int d q_{4} A\left(z_{4}, q_{4}\right) \Psi\left(z ; q_{4}\right)
$$

will define fields $\Psi(z)$ depending on four complex variables $z$, and the relevant functions will contain the Euclidean invariant form $z^{2}=z_{1}^{2}+z_{2}^{2}$ $+z_{3}^{2}+z_{4}^{2}$. The interaction kernel in $z$-space which describes local $q$-space interactions will be

$$
W^{(4)}\left(z, z^{\prime}, z^{\prime \prime}\right)=\int d^{4} q A^{(4)}(z, q) A^{(4)}\left(z^{\prime}, q\right) A^{(4)}\left(z^{\prime \prime}, q\right),
$$

where $d^{4} q=d^{3} q d q_{4}$ and $A^{(4)}(z, q)=A^{(3)}(z, q) A\left(z_{4}, q_{4}\right)$. Integration gives

$W^{(4)}\left(z, z^{\prime}, z^{\prime \prime}\right)=\left(\pi\left(\frac{3}{2} a\right)^{2}\right)^{-1} \exp \left[-\frac{\left(z-z^{\prime}\right)^{2}+\left(z^{\prime}-z^{\prime \prime}\right)^{2}+\left(z^{\prime \prime}-z\right)^{2}}{6 a^{2}}\right]$.

With $d^{4} \mu(z)=d^{3} \mu(z) d \mu\left(z_{4}\right)$ and

$$
\begin{aligned}
& -\boldsymbol{\nabla}=\gamma_{1} \frac{\partial}{\partial z_{1}}+\cdots+\gamma_{4} \frac{\partial}{\partial z_{4}} \\
& -\square_{z}=\frac{\partial^{2}}{\partial z_{1}^{2}}+\cdots+\frac{\partial^{2}}{\partial z_{4}^{2}},
\end{aligned}
$$

where $\gamma_{1}, \ldots, \gamma_{4}$ are anti-Hermitian, the Euclidean equations corresponding to Eqs. $(20 \mathrm{a}, \mathrm{b}, \mathrm{c})$ can be transformed into the form

$$
\begin{aligned}
& \left(i \boldsymbol{V}_{z}+m\right) \Psi(z) \\
& \quad+g \iint d^{4} \mu\left(z^{\prime}\right) d^{4} \mu\left(z^{\prime \prime}\right) W^{(4)}\left(z, \bar{z}^{\prime}, \bar{z}^{\prime \prime}\right) \Phi\left(z^{\prime}\right) \Psi\left(z^{\prime \prime}\right)=0 \\
& \bar{\Psi}(\bar{z})\left(-i \boldsymbol{V}_{z}+m\right) \\
& \quad+g \iint d^{4} \mu\left(z^{\prime}\right) d^{4} \mu\left(z^{\prime \prime}\right) W^{(4)}\left(\bar{z}, \bar{z}^{\prime}, z^{\prime \prime}\right) \bar{\Psi}\left(\bar{z}^{\prime \prime}\right) \Phi\left(z^{\prime}\right)=0 \\
& \left(\square_{z}+x^{2}\right) \Phi(z) \\
& \quad-g \iint d^{4} \mu\left(z^{\prime}\right) d^{4} \mu\left(z^{\prime \prime}\right) W^{(4)}\left(z, z^{\prime}, \bar{z}^{\prime \prime}\right) \Psi\left(\bar{z}^{\prime}\right) \Psi\left(z^{\prime \prime}\right)=0
\end{aligned}
$$

The same equations could be dirived from an action integral in 4-dimensional $z$-space, obtained by transforming the action integral of Euclidean $q$-space

$$
\begin{aligned}
I=-\int d^{4} q \bar{\Psi}[q]\left(\boldsymbol{V}_{q}+m\right) \Psi[q] & -\frac{1}{2} \int d^{4} q\left\{\frac{\partial \Phi[q]}{\partial q_{\mu}} \frac{\partial \Phi[q]}{\partial q_{\mu}}+x^{2} \Phi^{2}[q]\right\} \\
& -g \int d^{4} q \Phi[q] \Psi[q] \Psi[q]
\end{aligned}
$$


into $z$-space, that is from

$$
\begin{aligned}
I= & -\int d^{4} \mu(z) \bar{\Psi}(\bar{z})\left(\nabla_{z}+m\right) \Psi(z) \\
& -\frac{1}{2} \int d^{4} \mu(z)\left\{-\Phi(\bar{z}) \nabla_{z}^{2} \Phi(z)+x^{2} \Phi(\bar{z}) \Phi(z)\right\} \\
& -g \iiint d^{4} \mu(z) d^{4} \mu\left(z^{\prime}\right) d^{4} \mu\left(z^{\prime \prime}\right) W^{(4)}\left(\bar{z}, z^{\prime}, \bar{z}^{\prime \prime}\right) \Phi(z) \bar{\Psi}\left(\bar{z}^{\prime}\right) \Psi\left(z^{\prime \prime}\right) .
\end{aligned}
$$

At least within the framework of a $c$-number theory, the equations

$$
\frac{\delta I}{\delta \bar{\Psi}(\bar{z})}=0, \quad \frac{\delta I}{\delta \Psi(z)}=0, \quad \frac{\delta I}{\delta \Phi(\bar{z})}=0
$$

would give $(22 \mathrm{a}, \mathrm{b}, \mathrm{c})$. This assumes, however, a variational calculus in which variations are restricted to analytic functions of the variables $z$ with the reproducing kernel $U^{(4)}\left(z-\bar{z}^{\prime}\right)$. That is, variations $\delta \Phi(z)$ of the function $\Phi(z)$ have to satisfy the identity

$$
\delta \Phi(z)=\int d^{4} \mu\left(z^{\prime}\right) U^{(4)}\left(z-\bar{z}^{\prime}\right) \delta \Phi\left(z^{\prime}\right)
$$

and the unit kernel $U^{(4)}\left(z-\bar{z}^{\prime}\right)=U^{(3)}\left(z-\bar{z}^{\prime}\right) U\left(z_{4}-\bar{z}_{4}^{\prime}\right)$ appears instead of the $\delta$-function in expressions like

$$
\frac{\delta \Phi(z)}{\delta \Phi\left(z^{\prime}\right)}=U^{(4)}\left(z-\bar{z}^{\prime}\right)
$$

Starting from an action integral in $z$-space, the Green's function equations of quantum field theory can be obtained by means of a simple formal quantization with the help of external sources $[9,10]$, without reference to $q$-space. In introducing analytic source functions $\bar{\xi}(\bar{z}), \eta(z)$, $J(z)$, where $\bar{\xi}, \eta$ are anti-commuting quantities, and replacing the action integral $I$ by

$$
I^{\prime}=I+\int d^{4} \mu(z)\{\bar{\xi}(\bar{z}) \Psi(z)+\bar{\Psi}(\bar{z}) \eta(z)+\Phi(\bar{z}) J(z)\},
$$

the corresponding $c$-number field equations would have in addition to the terms of the Eqs. (23c) additional inhomogeneous source terms. The generating functional

$$
\chi=\chi[\xi, \eta, J]
$$

of the Green's functions satisfies the equations obtained by applying the inhomogeneous equations on $\chi$, after replacing the fields by functional differential operators according to

$$
\Psi(z) \rightarrow i \frac{\delta}{\delta \bar{\xi}(\bar{z})}, \quad \bar{\Psi}(\bar{z}) \rightarrow \frac{1}{i} \frac{\delta}{\delta \eta(z)}, \quad \Phi(z) \rightarrow i \frac{\delta}{\delta J(\bar{z})} .
$$


One obtains the equations

$$
\begin{gathered}
\left\{\left(i \boldsymbol{\nabla}_{z}+m\right) i \frac{\delta}{\delta \bar{\xi}(\bar{z})}-g \iint d^{4} \mu\left(z^{\prime}\right) d^{4} \mu\left(z^{\prime \prime}\right) W^{(4)}\left(z, \bar{z}^{\prime}, \bar{z}^{\prime \prime}\right) \frac{\delta}{\delta J\left(\bar{z}^{\prime}\right)} \frac{\delta}{\delta \bar{\xi}\left(\bar{z}^{\prime \prime}\right)}\right. \\
-\eta(z)\} \chi[\bar{\xi}, \eta, J]=0, \\
\left\{\frac{1}{i} \frac{\delta}{\delta \eta(z)}\left(i \nabla_{z}+m\right)+g \iint d^{4} \mu\left(z^{\prime}\right) d^{4} \mu\left(z^{\prime \prime}\right) W^{(4)}\left(\bar{z}, \bar{z}^{\prime}, z^{\prime \prime}\right) \frac{\delta}{\delta \eta\left(z^{\prime}\right)} \frac{\delta}{\delta J\left(\bar{z}^{\prime \prime}\right)}\right. \\
\quad-\bar{\xi}(\bar{z})\} \chi[\bar{\xi}, \eta, J]=0, \\
\left\{\left(\square_{z}+x^{2}\right) i \frac{\delta}{\delta J(\bar{z})}-g \iint d^{4} \mu\left(z^{\prime}\right) d^{4} \mu\left(z^{\prime \prime}\right) W^{(4)}\left(z, z^{\prime}, \bar{z}^{\prime \prime}\right) \frac{\delta}{\delta \eta\left(z^{\prime}\right)} \frac{\delta}{\delta \bar{\xi}\left(\bar{z}^{\prime \prime}\right)}\right. \\
-J(z)\} \chi[\bar{\xi}, \eta, J]=0 .
\end{gathered}
$$

If one expands $\chi[\xi, \eta, J]$ in a power series

$$
\begin{aligned}
\chi[\xi, \eta, J]= & \sum_{f^{\prime}, f^{\prime \prime}, f} \frac{(-i)^{f^{\prime+f^{\prime \prime+f}}}}{f^{\prime} ! f^{\prime \prime} ! f !} \int \cdots \int d \mu\left(z_{(1)}^{\prime}\right) \ldots d \mu\left(z_{\left(f^{\prime}\right)}^{\prime}\right) \\
& \cdot d^{4} \mu\left(z_{(1)}^{\prime \prime}\right) \ldots d^{4} \mu\left(z_{\left(f^{\prime \prime}\right)}^{\prime \prime}\right) d^{4} \mu\left(z_{(1)}\right) \ldots d^{4} \mu\left(z_{(f)}\right) \\
& \left.\cdot \xi\left(\bar{z}_{(1)}^{\prime}\right) \ldots \bar{\xi}_{\left(\bar{z}^{\prime}\right)}^{\prime}\right) G\left(z_{(1)}^{\prime}, \ldots, z_{\left(f^{\prime}\right)}^{\prime} ; \bar{z}_{(1)}, \ldots, \bar{z}_{(f)} ; \bar{z}_{(1)}^{\prime \prime}, \ldots, \bar{z}_{\left(f^{\prime \prime}\right)}^{\prime \prime}\right) \\
& \cdot \eta\left(z_{\left(f^{\prime \prime}\right)}^{\prime \prime}\right) \ldots \eta\left(z_{(1)}^{\prime \prime}\right) J\left(z_{(1)}\right) \ldots J\left(z_{(f)}\right)
\end{aligned}
$$

the Eqs. (26a, b, c) define the coupled equation system of the Green's functions $G$. With the help of simple identities, some of the variables $\bar{z}$ related to the sources $J(z)$ can be changed into variables $z$, according to convenience. The functions $G$ are simple $z$-transforms of the corresponding time-ordered Green's functions in Euclidean $q$-space.

The relationship between Green's functions and scattering amplitudes is similar to that in $q$-space. In quantum electrodynamics, the Green's function equations and their perturbative solution have been studied in some detail [11]. In terms of Fourier representations, the expressions obtained are almost identical to those in $q$-space, due to the simple relationship corresponding to $(10 \mathrm{a}, \mathrm{b}, \mathrm{c})$. In $z$-space, on the other hand, the equations contain the non-local kernel $W^{(4)}\left(z, z^{\prime}, z^{\prime \prime}\right)$, and a theory corresponding to local $q$-space interactions can be considered as embedded in a theory with more general $W^{(4)}$ kernels. One simple modification of $W^{(4)}$ which has been studied leads to finite self-energy and vertex expressions and, in the limit, to a simple regularisation of electrodynamics. This should be reported by one of the authors (D.K.S.) in a separate paper. 


\section{Free Particle Propagators}

The effect of the analytic representation can be exhibited explicitly in the case of the free particle propagators. For a scalar field, the free one-particle Green's function $\triangle\left(z, \bar{z}^{\prime}\right)$ satisfies the equation

$$
\left(\square_{z}+x^{2}\right) \triangle\left(z, \bar{z}^{\prime}\right)=U^{(4)}\left(z-\bar{z}^{\prime}\right) .
$$

The solution of the corresponding equation for a spin $1 / 2$ field,

$$
\left(i \boldsymbol{\nabla}_{z}+m\right) S\left(z, \bar{z}^{\prime}\right)=U^{(4)}\left(z-\bar{z}^{\prime}\right)
$$

can be constructed from $\triangle\left(z, \bar{z}^{\prime}\right)$ by writing

$$
S\left(z, \bar{z}^{\prime}\right)=\left(-i \boldsymbol{\nabla}_{z}+m\right) \triangle_{x=m}\left(z, \bar{z}^{\prime}\right) .
$$

As in $q$-space [12-14], a convenient parametric integral form of $\triangle\left(z, \bar{z}^{\prime}\right)$ can be obtained by introducing a "proper-time parameter" $s$, and solving the equation

$$
i \frac{\partial}{\partial s} \triangle\left(z, \bar{z}^{\prime} ; s\right)=\left(\square_{z}+\varkappa^{2}\right) \triangle\left(z, \bar{z}^{\prime} ; s\right)
$$

with the initial condition

$$
\triangle\left(z, \bar{z}^{\prime} ; s=0\right)=U^{(4)}\left(z-\bar{z}^{\prime}\right) .
$$

Proceeding in a similar way as in $q$-space, one finds

$$
\triangle\left(z, \bar{z}^{\prime} ; s\right)=\frac{a^{4}}{\left(a^{2}+i s\right)^{2}} \exp \left[-\frac{1}{4} \frac{\left(z-\bar{z}^{\prime}\right)^{2}}{a^{2}+i s}-i \varkappa^{2} s\right] .
$$

A Green's function solution of Eq. (27a) is given by

$$
\triangle\left(z, \bar{z}^{\prime}\right)=i \int_{0}^{\infty} d s \triangle\left(z, \bar{z}^{\prime} ; s\right) .
$$

As in Euclidean $q$-space, and in contrast to Minkowski $q$-space, the integral $i \int_{-\infty}^{0} d s \triangle\left(z, \bar{z}^{\prime} ; s\right)$ defines the same Green's function. Apart from a factor, the integrand (28c) would reduce to the corresponding $q$-space function for $a^{2}=0$. Its essential singularity has been deplaced from $s=0$ to $s=i a^{2}$ in the complex $s$-plane and is avoided by the integration path.

The integration can be directly performed for zero rest mass, $x=0$. In writing $D\left(z, \bar{z}^{\prime}\right)$ for the function $\triangle\left(z, \bar{z}^{\prime}\right)$ for $\varkappa=0$, and introducing the notation

one has

$$
X=\left(z-\bar{z}^{\prime}\right)^{2},
$$

$$
D\left(z, \bar{z}^{\prime}\right)=D(X),
$$


with

$$
\begin{aligned}
D(X) & =i \int_{0}^{\infty} d s \frac{a^{4}}{\left(a^{2}+i s\right)^{2}} \exp \left[-\frac{1}{4} \frac{X}{a^{2}+i s}\right] \\
& =\int_{a^{2}}^{\infty} d w \frac{a^{4}}{w^{2}} \exp \left[-\frac{X}{4 w}\right]=a^{2} \int_{0}^{1} d v \exp \left[-\frac{X}{4 a^{2}} v\right],
\end{aligned}
$$

or

$$
D(X)=-\frac{4 a^{4}}{X}\left\{\exp \left[-\frac{X}{4 a^{2}}\right]-1\right\} .
$$

This is an analytic function of $z$ and $\bar{z}^{\prime}$ for all finite values of the complex variables.

For $x \neq 0$, with the expressions $(28 \mathrm{c}, \mathrm{d})$, one still has

$$
\triangle\left(z, \bar{z}^{\prime}\right)=\triangle(X)
$$

and from the Eq. (27a) one derives the differential equation

$$
\left\{\left(2+X \frac{d}{d X}\right) 4 \frac{d}{d X}-x^{2}\right\} \triangle(X)=-\exp \left[-\frac{X}{4 a^{2}}\right] .
$$

For $X=0$, the expression $(28 \mathrm{c}, \mathrm{d})$ gives the finite value

where

$$
\begin{aligned}
\triangle(X & =0)=i \int_{0}^{\infty} d s \frac{a^{4}}{\left(a^{2}+i s\right)^{2}} \exp \left[-i \varkappa^{2} s\right] \\
& =\varkappa^{2} a^{4} \exp \left[\varkappa^{2} a^{2}\right] \Gamma\left(-1, \varkappa^{2} a^{2}\right),
\end{aligned}
$$

$$
\Gamma\left(-1, \varkappa^{2} a^{2}\right)=\int_{\varkappa^{2} a^{2}}^{\infty} d y \frac{\exp [-y]}{y^{2}}
$$

is the incomplete $\Gamma$-function. In the limit of large real positive $X$, the same expression gives

$$
\lim _{X \rightarrow \infty} \triangle(X)=0
$$

The solution of the differential Eq. $(30 \mathrm{~b})$, with the boundary conditions $(30 \mathrm{c}, \mathrm{e})$, can be constructed for real $X$ by standard methods [15].

If one multiplies the Eq. $(30 \mathrm{~b})$ by $X^{1 / 2}$, it is transformed into the Sturm-Liouville form

$\left\{\frac{d}{d X}\left(4 X \frac{d}{d X}\right)-\frac{1}{X}-x^{2}\right\}\left(X^{1 / 2} \triangle(X)\right)=-X^{1 / 2} \exp \left[-\frac{X}{4 a^{2}}\right]$,

and the boundary conditions become

$$
X^{1 / 2} \triangle(X)=0 \text { for } X=0 \text { and } X=\infty .
$$

The corresponding homogeneous equation,

$$
\left\{\frac{d}{d X}\left(4 X \frac{d}{d X}\right)-\frac{1}{X}-\varkappa^{2}\right\} g(X)=0
$$


has the two independent Bessel function solutions

$$
\begin{gathered}
g_{0}(X)=I_{1}\left(\varkappa X^{1 / 2}\right), \\
g_{\infty}(X)=K_{1}\left(\varkappa X^{1 / 2}\right),
\end{gathered}
$$

which satisfy the boundary conditions

$$
g_{0}(0)=0, \quad g_{\infty}(X=\infty)=0 .
$$

The Wronskian of the two functions is $-1 / X$ which, combined with the factor $4 X$ in the first term of the differential Eqs. (31 a), (32a), leads to the Green's function

$$
G(X, Y)=\left\{\begin{array}{lll}
\frac{1}{4} I_{1}\left(\varkappa X^{1 / 2}\right) K_{1}\left(\varkappa Y^{1 / 2}\right) & & X \leqq Y \\
\frac{1}{4} K_{1}\left(\varkappa X^{1 / 2}\right) I_{1}\left(\varkappa Y^{1 / 2}\right) & \text { for } & X \geqq Y .
\end{array}\right.
$$

The corresponding solution of the inhomogeneous differential Eq. $(30 \mathrm{~b})$ is accordingly

with

$$
\triangle(X)=C_{0}(X) K_{1}\left(\varkappa X^{1 / 2}\right)+C_{\infty}(X) I_{1}\left(\varkappa X^{1 / 2}\right)
$$

$$
\begin{aligned}
& C_{0}(X)=\frac{1}{4 X^{1 / 2}} \int_{0}^{X} d Y I_{1}\left(x Y^{1 / 2}\right) Y^{1 / 2} \exp \left[-\frac{Y}{4 a^{2}}\right], \\
& C_{\infty}(X)=\frac{1}{4 X^{1 / 2}} \int_{X}^{\infty} d Y K_{1}\left(x Y^{1 / 2}\right) Y^{1 / 2} \exp \left[-\frac{Y}{4 a^{2}}\right] .
\end{aligned}
$$

The solution can be checked to satisfy the boundary conditions (30c, e). Since the integral representation $(28 \mathrm{c}, \mathrm{d})$ is valid for complex $X=\left(z-\bar{z}^{\prime}\right)^{2}$, the solution can be extended to complex values of the argument. For $x=0$, the expression (33a) of $\triangle(X)$ can be shown to reduce to the expression $(29 \mathrm{~d})$ of $D(X)$. The Bessel function $K_{1}\left(x X^{1 / 2}\right)$ is singular at $X=0$, but the function $\triangle(X)$ has been constructed using the boundary condition that it should be finite at that point. The two terms of the expression (33a) of $\triangle(X)$ are multi-valued functions, but the sum of the two terms gives a single-valued analytic function for all finite values of $X$. These last points have been investigated in detail by Dr. R. B. Jones.

The authors would like to express their thanks to Dr. R. B. Jones for helpful discussions and contributions. 


\section{References}

1. Bargmann, V.: Commun. Pure Appl. Math. 14, 187, (1961); 20, 1 (1967).

2. - Proc. Natl. Acad. Sci. U.S. 48, 199 (1962).

3. Glauber, R. J.: Phys. Rev. 131, 2766 (1963); Quantum optics and electronics. New York: Gordon and Breach 1965.

4. Klaudder, J. R.: J. Math. Phys. 4, 1055,1058 (1963); 5, 177 (1964). Klauder, J. R., and J. McKenna: J. Math. Phys. 5, 878 (1964); 6, 68 (1965). - Schweber, S. S.: J. Math. Phys. 3, 831 (1962).

5. Schwinger, J.: Phys. Rev. 115, 721 (1959).

6. Nakano, T.: Progr. Theor. Phys. 21, 241 (1959).

7. Symanzik, K.: In: Analysis in function space, Ed. W. T. Martin and I. Segal, p. 197. Cambridge, Mass.: MTT Press 1964; J. Math. Phys. 7, 510 (1966).

8. v. Neumann, J.: Mathematische Grundlagen der Quantenmechanik, S. 123. Berlin: Springer 1932.

9. Symanzik, K.: Funktionale in der Feldtheorie, Thesis, Göttingen (1954); J. Math. Phys. 1, 249 (1960).

10. Valatin, J. G.: Proc. Roy. Soc. (A), 229, 221 (1955).

11. Saraswati, D. K.: Ph. D. Thesis, University of London (1968), (unpublished).

12. Fock, V.: Phys. Z. Sowjet. 12, 404 (1937).

13. Schwinger, J.: Phys. Rev. 82, 664 (1951).

14. Valatin, J. G.: Proc. Roy. Soc. (A) 222, 93 (1954).

15. Courant, R., and D. Hilbert: Methods of mathematical physics. Vol. I, p. 351. New York: Interscience Publishers 1953.

D. K. Saraswati

Physics Department

Notre Dame University

Nelson, British Columbia
J. G. Valatin

Department of Physics

Queen Mary College

Mile End Road

London E. 1., England 